\title{
Correction to: Association Between Diethylhexyl Phthalate Exposure and Thyroid Function: A Meta-Analysis by Kim MJ, Moon S, Oh B-C, Jung D, Choi K, and Park YJ. Thyroid 2019;29:183-192. DOI: 10.1089/thy.2018.0051
}

T the February 2019 issue of Thyroid (vol. 29, no. 2; 183-192) the article entitled "Association Between Diethylhexyl

Phthalate Exposure and Thyroid Function: A Meta-Analysis" by Kim et al. requires correction.

Figure legends 2-4 mention PFOS, PFOA, and PFHxS instead of MEHP, MEHHP, and MEOHP as discussed in the article. The legends originally read:

FIG. 2. Forest plots of the correlation coefficient with corresponding confidence intervals (CIs) for the correlation between perfluorooctane sulfonate (PFOS) and thyroid hormone. (A) Correlation between PFOS and thyrotropin (TSH). (B) Correlation between PFOS and free thyroxine (fT4). (C) Correlation between PFOS and total thyroxine (TT4).

FIG. 3. Forest plots of the correlation coefficient with corresponding CIs for the correlation between perfluorooctanoic acid (PFOA) and thyroid hormone. (A) Correlation between PFOA and TSH. (B) Correlation between PFOA and fT4. (C) Correlation between PFOA and TT4.

FIG. 4. Forest plots of the correlation coefficient with corresponding CIs for the correlation between perfluorohexane sulfonic acid (PFHxS) and thyroid hormone. (A) Correlation between PFHxS and TSH. (B) Correlation between PFHxS and fT4. (C) Correlation between PFHxS and TT4.

The legends should read:

FIG. 2. Forest plots of the correlation coefficient with corresponding confidence intervals (CIs) for the correlation between monoethylhexyl phthalate (MEHP) and thyroid hormone. (A) Correlation between MEHP and thyrotropin (TSH). (B) Correlation between MEHP and free thyroxine (fT4). (C) Correlation between MEHP and total thyroxine (TT4).

FIG. 3. Forest plots of the correlation coefficient with corresponding CIs for the correlation between mono (2-ethyl-5hydroxyhexyl) phthalate (MEHHP) and thyroid hormone. (A) Correlation between MEHHP and TSH. (B) Correlation between MEHHP and fT4. (C) Correlation between MEHHP and TT4.

FIG. 4. Forest plots of the correlation coefficient with corresponding CIs for the correlation between mono (2-ethyl-5oxohexyl) phthalate (MEOHP) and thyroid hormone. (A) Correlation between MEOHP and TSH. (B) Correlation between MEOHP and fT4. (C) Correlation between MEOHP and TT4.

The online version has been corrected to reflect this. The authors apologize for these errors. 